\title{
Monoclonal Antibodies Reveal Cell-Type-Specific Antigens in the Sexually Dimorphic Olfactory System of Manduca sexta. II. Expression of Antigens During Postembryonic Development
}

\author{
Akira Hishinuma, ${ }^{1, a}$ Susan Hockfield, ${ }^{2, b}$ Ronald McKay, ${ }^{2, c}$ and John G. Hildebrand ${ }^{1, a}$ \\ 'Department of Biological Sciences, Columbia University, New York, New York 10027, and ${ }^{2}$ Cold Spring Harbor \\ Laboratory, Cold Spring Harbor, New York 11724
}

Two classes of monoclonal antibodies specific to the olfactory system of Manduca sexta have been isolated: the olfactory-specific antibody (OSA), which specifically recognizes many or all olfactory receptor cells (ORCs) in both males and females, and the male olfactory-specific antibody (MOSA), which stains male-specific receptor cells (principally or exclusively sex-pheromone receptors present only in antennae of males; Hishinuma et al., 1988). In the investigation reported here, we examined the expression of the antigens during postembryonic development in order to correlate the presence of particular antigens with the status of differentiation of the ORCs or with their acquisition of particular functions.

As assessed immunocytochemically, the OSA recognizes certain epithelial cells in the antennal imaginal disk of the fifth-instar larva. Later, during the first $70 \mathrm{hr}$ of adult development, when differentiative cell divisions are occurring in the antennal epithelium to generate ORCs and the other cells that make up olfactory sensilla, no cells are stained. Immediately after this period of mitoses, the OSA immunoreactivity reappears exclusively in the ORCs, which begin to elaborate axons as an early event in their differentiation. On immunoblots, the OSA recognizes specific sets of molecules (distinguished on the basis of their apparent molecular weights): 53,000 and $59,000 \mathrm{Da}$ antigens in the disk epithelial cells in the last-instar larva; $53,000,59,000$, and $66,000 \mathrm{Da}$ antigens in the ORCs from 15 to $60 \%$ of metamorphic adult development; and $42,000,59,000$, and $66,000 \mathrm{Da}$ antigens in the ORCs from 60 to $100 \%$ of adult development.

The MOSA also recognizes a subset of the epithelial cells

\footnotetext{
Received Apr. 16, 1987; revised June 23, 1987; accepted June 24, 1987

We dedicate this paper to the memory of our colleague Dr. Stephen Schuetze, who generously gave us much help and encouragement in this project.

We are grateful to E. Waldvogel, C. Bautista, R. A. Montague, E. Slattery, and $N$. Antinoro for expert technical assistance in various aspects of this research; Drs. S. Hoskins, U. Homberg, and M. Stengl for their critical reading of the manuscript; Drs. L. Eckhardt, T. Kingan, and C. Squires for helpful discussions; Drs. J. Buckner and J. Svoboda of the U.S.D.A. for generously supplying Manduca eggs; and S. Garner for preparing the typescript. This research was supported by NSF Grants BNS 83-12769 and BNS 86-41145 (to J.G.H.) and BNS 84-19240 (to S.H.), and NIH Grants 1R01 EY 06511 (to S.H.) and 1R01 NS 17556 (to R.M.). S.H. is a Klingenstein Fellow.

Correspondence should be addressed to John G. Hildebrand, ARL Division of Neurobiology, University of Arizona, 611 Gould-Simpson Science Building, Tucson, AZ 85721 .

- Present address: ARL Division of Neurobiology, University of Arizona, Tucson, AZ 85721 .

b Present address: Section of Neuroanatomy, Yale University School of Medicine, New Haven, CT 06510.

' Present address: MIT, Whitaker College, Cambridge, MA 02139.

Copyright (C) 1988 Society for Neuroscience $0270-6474 / 88 / 010308-08 \$ 02.00 / 0$
}

in the antennal disks in male and female larvae. After disappearing at the pupal molt, MOSA staining is not detected again in the epithelium of the developing male antenna until late in metamorphosis, when electroantennogram responses to female sex pheromones are first detectable. This correlation suggests that the MOSA antigen is expressed during the final maturation of the male-specific receptor cells and may be involved in some way in the olfactory functions of those cells, particularly in the detection of the female sex pheromones.

The development of adult olfactory receptor cells (ORCs) in the antennae of Manduca sexta has been studied in detail (Sanes and Hildebrand, 1976b). ${ }^{3} \mathrm{H}$-Thymidine birthdating indicates that precursor cells in the epithelium of the antennal imaginal disk start to divide about $25 \mathrm{hr}$ after the larval-pupal molt, at the initiation of adult development, to give risc to olfactory sensilla comprising several different types of cells. For example, each male-specific sensillum trichodeum consists of 5 different types of cells: trichogen, tormogen, and " 2 " cells (which form the cuticular sensory hair and its socket and receptor lymph), a glia-like cell, and a pair of sex-pheromone-specific ORCs. Incorporation of labeled thymidine into these cells is complete by $60 \mathrm{hr}$ after the larval-pupal molt, and very soon thereafter the ORCs begin to elaborate axons as an early event in their differentiation (Sanes and Hildebrand, 1976b). Later, during adult development, shortly before emergence of the adult moth, the ORCs associated with these male-specific antennal sensilla become responsive to the female sex pheromones (Schweitzer et al., 1976).

As part of our continuing studies of the functional organization and development of the antennal olfactory system and of the molecular basis of neuronal diversity underlying the odorspecificity of the ORCs, we have produced monoclonal antibodies (Mabs) using standard hybridoma techniques (Köhler and Milstein, 1976), from spleen cells of mice immunized with extracts of antennal lobes (ALs) - the primary olfactory centers in the brain - from male Manduca sexta (Hishinuma et al., 1988). One of these Mabs, the olfactory-specific antibody (OSA), recognizes membrane-associated glycoproteins that are expressed exclusively in many or all ORCs in both males and females. Immunoblot analysis showed that the OSA recognizes proteins with apparent $M_{r} s$ of $42,000,59,000$, and $66,000 \mathrm{Da}$, and have suggested that the 59,000 Da protein is processed to the 66,000 Da protein during axonal transport. A second Mab, the male olfactory-specific antibody (MOSA), recognizes a cytoplasmic 

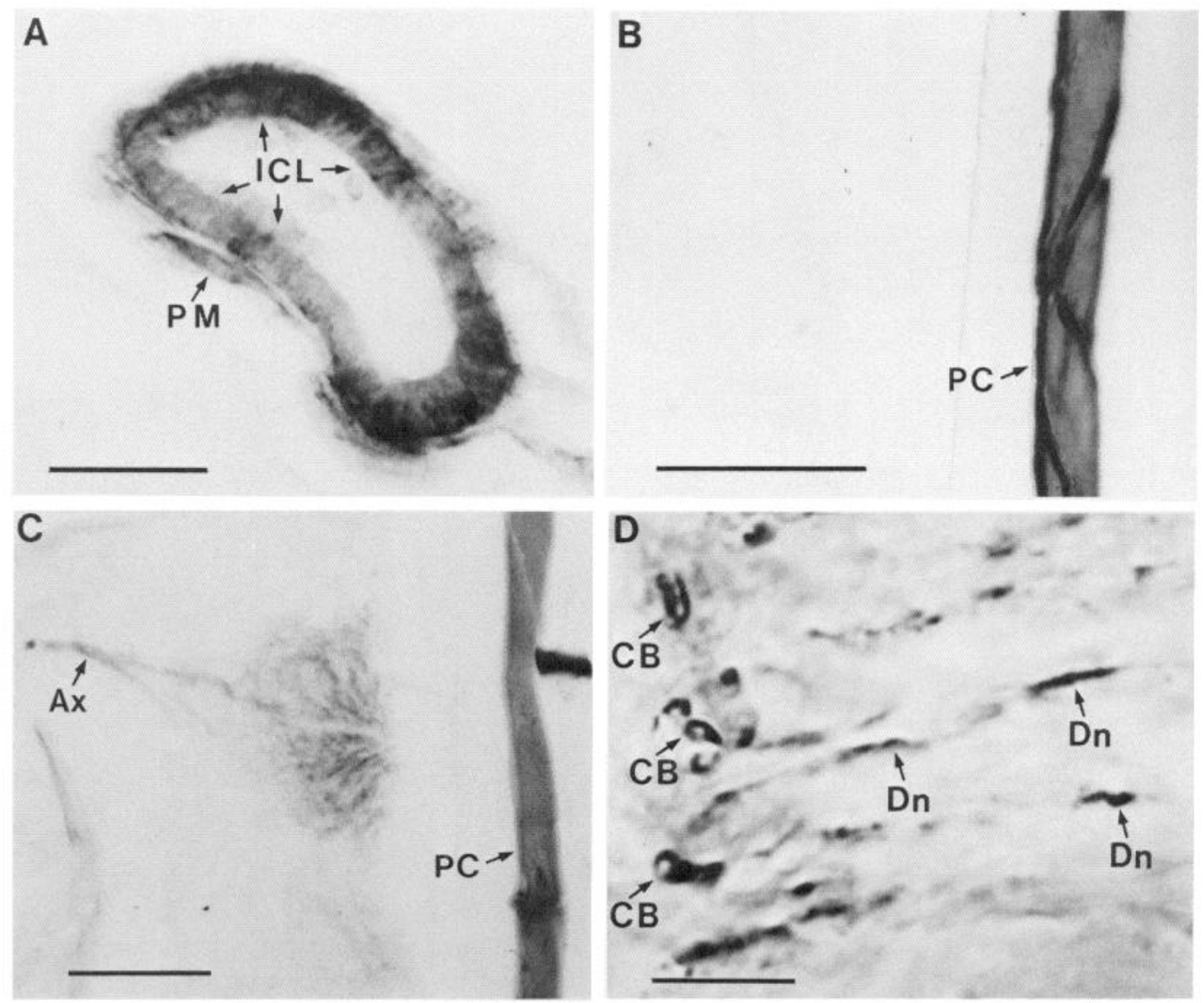

Figure 1. Immunostaining with the OSA during antennal development. A, Cross section of the proximal third of the antennal imaginal disk of the fifth-instar larva. Cells of the inner cell layer $(I C L)$ of the disk epithelium, as well as part of the peripodial membrane $(P M)$, are stained. $B-D$, Longitudinal sections (with the lumenal side of epithelium to the left and the apical side to the right, underlying the pupal cuticle (PC), which is visible in $B$ and $C$ but not $D$ ) of developing antennae at $3 \mathrm{hr}(B), 78 \mathrm{hr}(C)$, and stage $7(D)$ after the larval-pupal molt. At $3 \mathrm{hr}$ after the molt to the pupa $(B)$, no cells in the antenna are stained by the OSA. At $78 \mathrm{hr}$ after the molt $(C)$, clusters of ORCs in each presumptive annulus of the antennal flagellum are stained. Early in their differentiation, the ORCs send out axons, which form fascicles $(A x)$ and are also stained by the OSA. $D$, Immunostained ORC cell bodies $(C B)$ and dendrites $(D n)$ are evident at stage 7 , but no other types of cells are recognized by the OSA. Bars: $100 \mu \mathrm{m}(A) ; 20 \mu \mathrm{m}(B, C) ; 10 \mu \mathrm{m}(D)$.

antigen in certain ORCs present in the antennae of males, but not females. These male-specific ORCs are principally or exclusively specialized for the detection of the female sex pheromones (J. Hildebrand and K.-E. Kaissling, unpublished observations).

In this study, we examined the expression of the antigens during postembryonic development. The presence of OSA immunoreactivity reflects differentiation of the ORCs, and each of the electrophoretically distinct species of the OSA antigen is expressed at a characteristic stage of adult development. The MOSA antigen is first detectable at the late developmental stage, when antennal responses to the female sex pheromones are first detectable by electroantennography.

\section{Materials and Methods}

Manduca sexta (Lepidoptera: Sphingidae) were reared and staged by methods that have been described previously (Sanes and Hildebrand, 1976a; Tolbert et al., 1983). Staging of larvae during the last (fifth) instar was as described previously (Vince and Gilbert, 1977). Production of Mabs, immunocytochemistry, and immunoblots analysis has been described (Hishinuma et al., 1988). For immunoblots, tissue extract from 10 antennal imaginal disks or half of the antenna was loaded in each lane of a $10 \%$ polyacrylamide slab gel. Before stage 10 (about $60 \%$ of metamorphic adult development), when the adult cuticle has not yet tanned, the whole antenna was used. After stage 12, the antenna was cut open with iridectomy scissors, and pieces of the epithelium underlying the cuticle were isolated with fine forceps. The tissue was immediately homogenized in SDS sample buffer without a reducing agent ( $2 \%$ SDS, $5 \%$ glycerol, $0.001 \%$ bromphenol blue, $0.08 \mathrm{~m}$ Tris- $\mathrm{HCl}, \mathrm{pH}$ 6.8 ) in the presence of protease inhibitors ( $1 \mathrm{~mm}$ EDTA, $0.3 \mathrm{~mm}$ phenylmethylsulfonyl fluoride, $0.1 \mathrm{~mm}$ benzethonium $\mathrm{HCl}, 1 \mathrm{~mm}$ benzamidine, $5 \mu \mathrm{g} / \mathrm{ml}$ soybean trypsin inhibitor, $100 \mu \mathrm{g} / \mathrm{ml}$ bacitracin, $2 \mu \mathrm{g} /$ $\mathrm{ml}$ leupeptin; all from Sigma) (Shorr et al., 1981) by means of an allglass homogenizer on ice. The homogenate was boiled for $5 \mathrm{~min}$ and then centrifuged at $2000 \mathrm{rpm}$ (IEC HN-SII centrifuge) for $5 \mathrm{~min}$. After separation by PAGE (Laemmli, 1970), the protein bands were electrically transferred onto nitrocellulose sheets (Schleicher and Schuell) according to Towbin et al. (1979). The nitrocellulose sheets were pretreated with $3 \%$ bovine serum albumin (Sigma) in Tris-saline (10 mm Tris, 150 $\mathrm{mm} \mathrm{NaCl}, \mathrm{pH} 7.5$ ) with $0.05 \%$ Tween-20 (Sigma), and then incubated in the culture supernatant media (full strength) and subsequently in HRP-conjugated anti-mouse IgG (diluted 1:100; Boehringer Mannheim). The immunoreactive bands were visualized by treatment with 4-chloro-1-naphthol (Sigma) in the presence of $\mathrm{H}_{2} \mathrm{O}_{2}$.

\section{Results}

Antigens recognized by the OSA reflect differentiation of the ORCS

The antennal imaginal disk in a fifth-instar larva is a 2-layered, invaginated bulb. At the proximal tip, the 2 cell layers are con- 
Figure 2. Immunoblot analysis of the OSA antigens (apparent $M_{\mathrm{r}} \mathrm{s} 66,000$, $59,000,53,000$ and $42,000 \mathrm{Da}$ during metamorphic adult development. Five different gels $(A-E)$ are compared. $A$, OSA antigens extracted from the disk epithelium, as compared to those extracted from the developing antenna at stage 10 . Lane 1 , stage 10 antenna; lane 2 , fifth-instar antennal disks. $B-E$, Comparison of OSA antigens in extracts of stage 18 (pharate-adult) ALs (lane 3) with antigens in extracts of developing antennae at stages 2 (lane 4), 4 (lane 7), 5 (lane 5), 6 (lane 8), 7 (lane 6), 8 (lane 9), 10 (lanes 10,11, 13), 12 (lanes 12, 14), 16 (lane 15), and 18 (lane 16). Molecular-weight standards were pepsin $(34,700)$, egg albumin $(45,000)$, bovine serum albumin $(66,000)$, phosphorylase $(97,400)$, and $\beta$-galactosidase $(116,000)$.

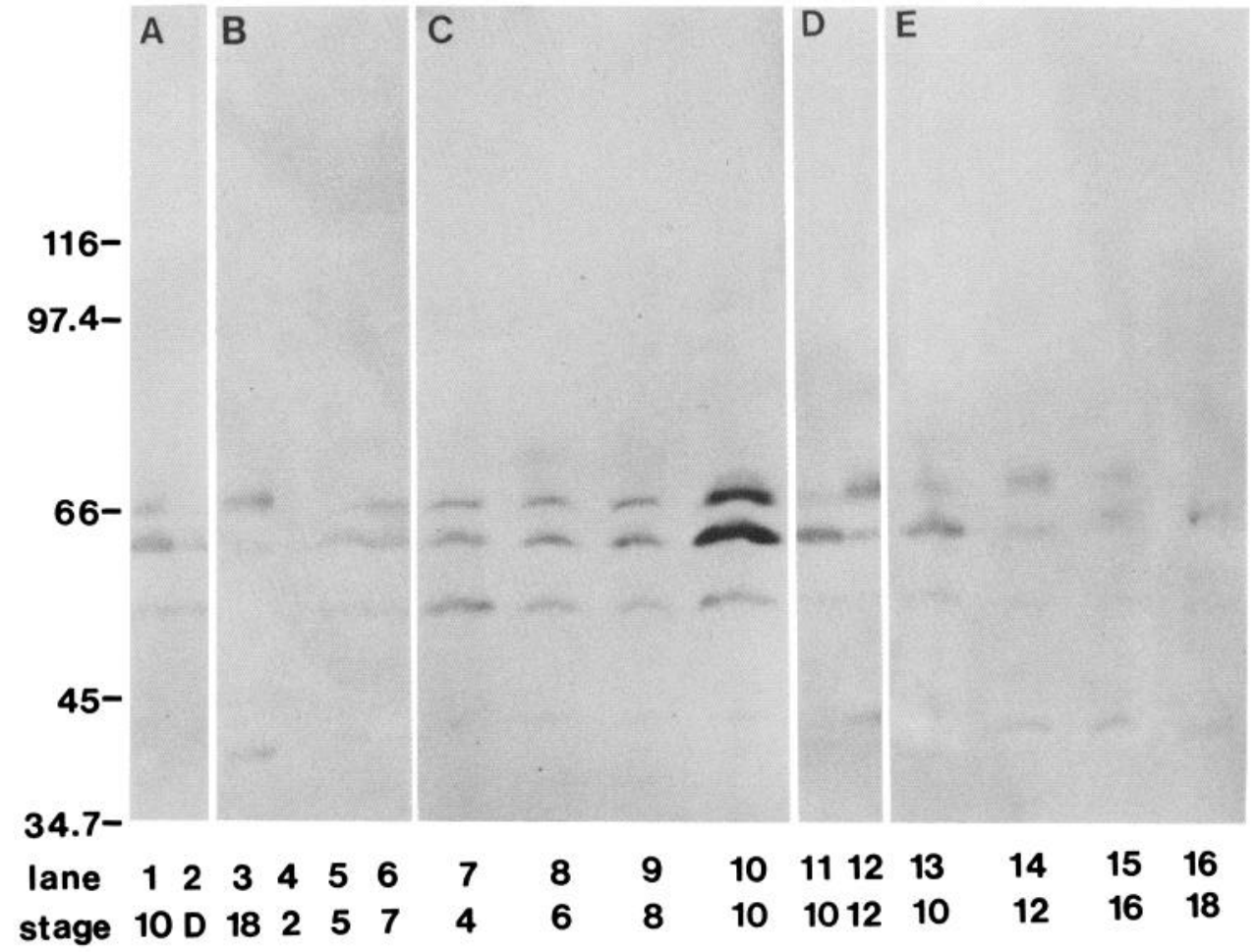

tinuous, and at the distal tip, the outer cell layer (the peripodial membrane; Eassa, 1953) is continuous with the epidermis of the larval head (Sanes and Hildebrand, 1976a). Most of the epithelial cells residing in the proximal two-thirds of the inner cell layer of the disk and the proximal one-third of the peripodial membrane are stained by the OSA in both males and females (Fig. 1A). Reconstruction of serial sections of 9 imaginal disks showed that the immunoreactive cells are evenly distributed in this region. The intensity of the OSA staining decreases gradually during the last $3 \mathrm{~d}$ (prepupal period) before the larvalpupal molt. The OSA immunoreactivity could not be detected in the everted antennal disk $3 \mathrm{hr}$ after the larval-pupal molt (Fig. 1B).

OSA immunoreactivity in the antennal epithelium was detected by light microscopy about $70 \mathrm{hr}$ after the larval-pupal molt, which corresponds to late stage 3 (of the 18 stages of adult development) (Table 1). We tested 22 animals (both males and females) between 60 and $85 \mathrm{hr}$ after the larval-pupal molt. The OSA reveals 2 clusters of nascent ORCs in each annulus of the developing antenna $70 \mathrm{hr}$ following the larval-pupal molt and

Table 1. Reappearance of OSA immunoreactivity in the ORCs in developing antennae

\begin{tabular}{lll} 
Hours after & Number of animals \\
\cline { 2 - 3 } pupal molt & OSA-negative & OSA-positive \\
\hline $60-64$ & 2 & 0 \\
$65-69$ & 4 & 0 \\
$70-74$ & 2 & 3 \\
$75-79$ & 1 & 4 \\
$80-85$ & 0 & 6 \\
\hline
\end{tabular}

a Twenty-four hours correspond to one stage up to stage 10 . before the interannular membranes are discernible (Fig. 1C). The clusters of OSA-positive ORCs in each annulus are aligned in 2 longitudinal rows along the length of the antenna. Staining with OSA confirms that the ORCs in each cluster send out axons in fascicles, which merge with the 2 main branches of the antennal nerve in the lumen of the antenna (Fig. 1C). Axonal outgrowth is one of the earliest events in the maturation of the ORCs (Wigglesworth, 1953; Sanes and Hildebrand, 1976b). Cell clusters lacking fascicles of axons are not stained by the OSA. At stage 7 of adult development, OSA-immunoreactive receptor cell bodies are found at the base of the antennal epithelium, and their immunoreactive dendrites traverse the epithelium (Fig. 1D).

PAGE (under nonreducing conditions) of extracts of antennal tissue (including cell bodies and segments of axons and dendrites of ORCs) from stage 18 pharate adults, followed by immunoblotting on nitrocellulose sheets, revealed OSA-immunoreactive bands of apparent $M_{\mathrm{r}} \mathrm{s} 42,000,59,000$, and 66,000 Da (Hishinuma et al., 1988). This analysis was extended to earlier stages in the postembryonic development of the antenna to examine whether any of the antigens expressed in pharate adults are also expressed in the epithelium of the imaginal disk, and whether the expression of any of the antigens is stage-specific. We used 10 antennal imaginal disks or halves of antennae in each lane in PAGE. Each sample was duplicated in 2 lanes, one for immunoblotting and the other for amido black staining, to verify that all samples included comparable amounts of protein.

The protein bands immunoreactive with the OSA during postembryonic development are summarized in Table 2. We homogenized the tissue in SDS sample buffer in the presence of a mixture of protease inhibitors immediately after dissection. The results were qualitatively identical on numerous repetitions. Because the intensity of immunoreactive bands was not quantified, we confined our discussion to the major bands. The OSA rec- 

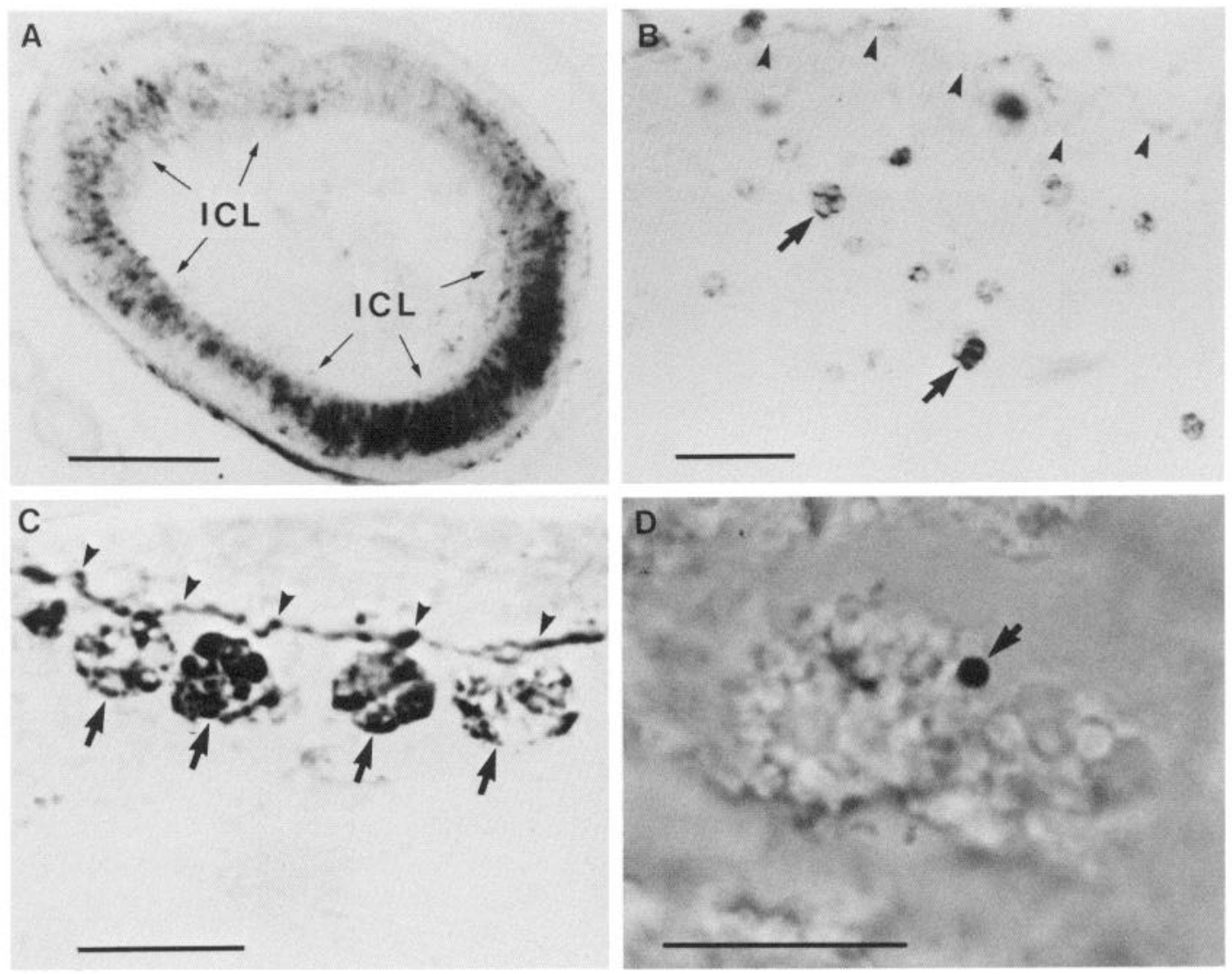

Figure 3. Immunostaining with the MOSA during antennal development. $A$, Cross section of the center of the antennal imaginal disk of a fifthinstar larva, showing staining of cells in the inner cell layer $(I C L)$ of the disk epithelium. $B-D$, Longitudinal sections of developing antennae at 3 $\mathrm{hr}$ after the larval-pupal molt $(B)$, stage $4(C)$, and stage $7(D)$. At $3 \mathrm{hr}$ after the pupal molt, the MOSA stains disperse cells in the lumen of the antenna outside the epithelium. Some of these cells form clusters (arrows in $B$ ). Some reaction product is also associated with the limiting membrane between the epithelium and the lumen (arrowheads). At stage 4, clusters of 20-30 MOSA-immunoreactive cells are found in the lumen adjacent to the epithelium (arrows in $C$ ). Again, reaction product is also associated with the limiting membrane (arrowheads). At stage 7, only a few of the clustered cells are immunoreactive (example at arrow in $D$ ). Bars: $50 \mu \mathrm{m}(A) ; 20 \mu \mathrm{m}(B-D)$.

ognizes 2 protein bands $(53,000$ and $59,000 \mathrm{Da})$ in the imaginal disk (Fig. 2A). In animals at $37 \mathrm{hr}$ after the larval-pupal molt, no bands were detected (Fig. $2 B$ ), which paralleled the immunocytochemical findings. In developing adults at stages 3-10, 3 major bands $(53,000,59,000$, and $66,000 \mathrm{Da})$ are recognized by the OSA (Fig. 2, B, C). Samples from the imaginal disks and the pupal antenna at stage 10 of adult development were applied in adjacent lanes, and the 53,000 and $59,000 \mathrm{Da}$ bands migrated to identical positions in both lanes (Fig. 2A). Another major change in the immunoreactive bands occurs between stages 10 and 12. The 53,000 Da band becomes faint, and a 42,000 Da band begins to be expressed to produce the adult pattern $(42,000$ 59,000 , and $66,000 \mathrm{Da}$ ) (Fig. 2D). Thus, the $59,000 \mathrm{Da}$ antigen in pharate adults is apparently also expressed in the imaginal disks. Comparison of relative intensities of the 59,000 and 66,000 Da bands from stages 12-18 shows that the $66,000 \mathrm{Da}$ band becomes increasingly faint in the antennal epithelium (Fig. $2 E$ ).

\section{The antigen recognized by the MOSA reflects functional maturation of the male-specific ORCs}

The MOSA stains certain epithelial cells in the antennal imaginal disks of both males and females (Fig. $3 A$ ). The immunoreactive cells are detected in the proximal two-thirds of the inner epithelial cell layer and in the proximal one-third of the peripodial membrane. The distribution of the stained cells is not uniform, but rather is more prominent in the proximal onethird of the inner cell layer than elsewhere. Staining intensity decreases during the prepupal period. At $3 \mathrm{hr}$ after the larval-

Table 2. Apparent molecular weights of the OSA-immunoreactive bands during metamorphic adult development

Developmental stage

Bands in immunoblots ${ }^{a}$ (molecular weight $\left.\times 1000\right)$

Fifth-instar imaginal disk

Developing adult, stages $3-10$

(72)

$\begin{array}{ccc} & 59 & 53 \\ 66 & 59 & 53 \\ 66 & 59 & (53)\end{array}$

Developing adult, stages $12-18$

${ }^{a}$ Minor bands are in parentheses. 

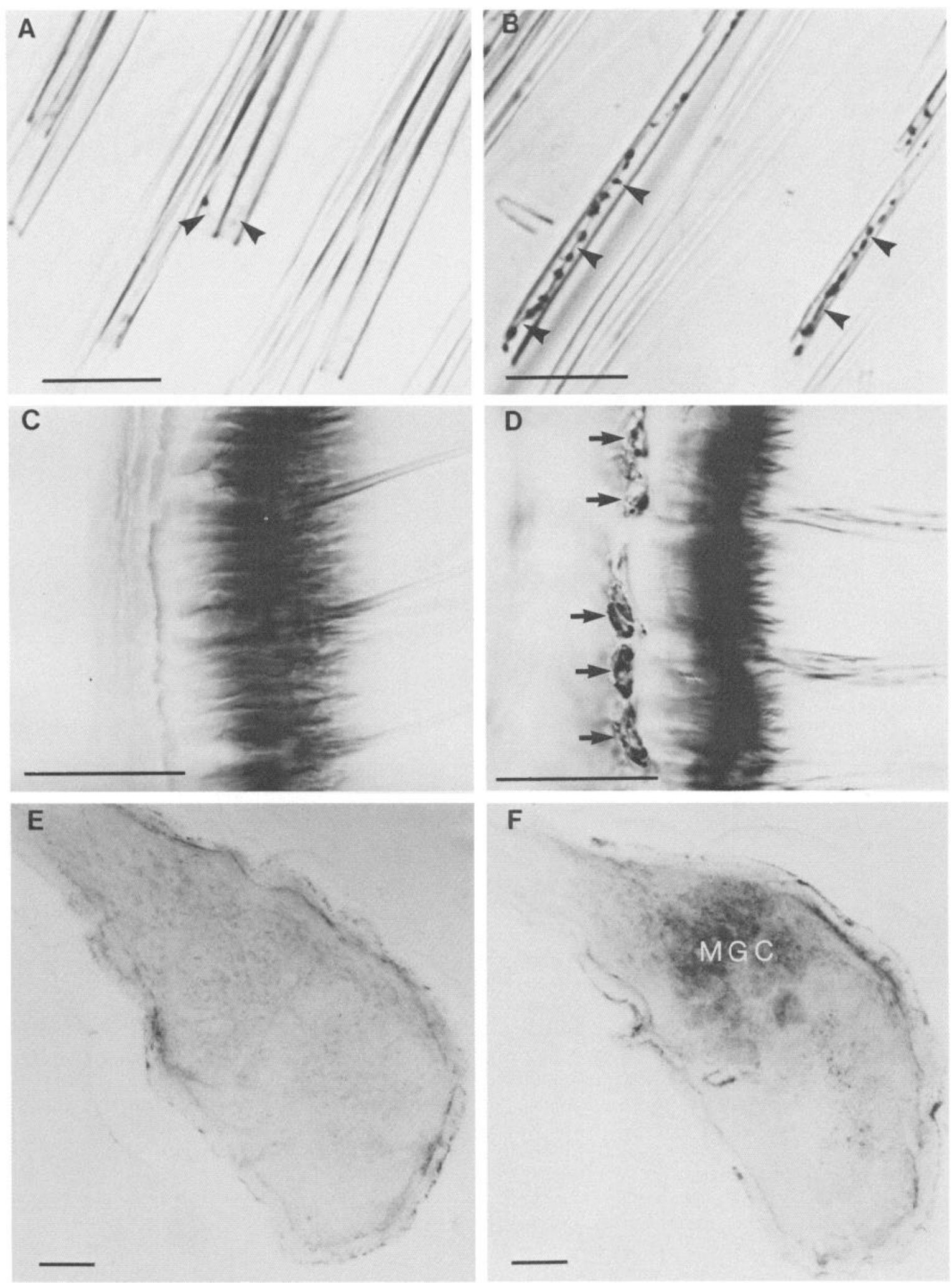

Figure 4. Immunostaining of male-specific sensilla trichodea $(A, B)$, cross sections of antennae $(C, D)$, and frontal sections of ALs $(E, F)$ from males at stages $16(A, C, E)$ and $17(B, D, F)$. Dendrites of the male-specific pheromone-receptor cells are stained by the MOSA at stage 17 (arrowheads in $B$ ), but only faintly at stage 16 (arrowheads in $A$ ). Similarly, receptor somata are stained by the MOSA at stage $17($ arrows in $D$ ), but not detectably at stage $16(C)$, and the MGC is stained at stage $17(F)$, but not at stage $16(E)$. Bars: $20 \mu \mathrm{m}(A, B) ; 100 \mu \mathrm{m}(C-F)$. 
pupal molt, no cells in the antennal epithelium are stained by the MOSA (data not shown). The MOSA stains another type of cell, different from the ORCs, in the early phases of postembryonic development in both males and females. These cells are spheroidal and never grow processes. At $3 \mathrm{hr}$ after the larvalpupal molt, they are scattered in the lumen of the developing antenna inside the antennal epithelium (Fig. $3 B$ ). Some of these cells form clusters of several cells. During the next few days they apparently multiply, becoming clusters of 20-30 cells by stage 4 (Fig. $3 C$ ). Every cell in each cluster is immunoreactive at stage 4; thereafter, the cells lose immunoreactivity to the MOSA. At stage 7 , the clusters are still identifiable in the phase-contrast microscope, but clusters that contain immunoreactive cells are very rare, and only a few cells in such clusters are immunoreactive (Fig. $3 D$ ). After the clusters comprise only nonimmunoreactive cells, they eventually disappcar by stage 16 of adult development.

The MOSA immunoreactivity appears in a subpopulation of the ORCs in the antennal epithelium of males, but not females, at the same time (stage 17) when electroantennograms first show responses to the female sex pheromones. Since our preliminary results indicated that MOSA immunoreactivity reappears at about stage 17 in male ORC cell bodies, we extended our immunocytochemical analysis to search for temporospatial gradients of expression. Frozen sections from the base, center, and tip of the antennal flagellum and from the ALs of the same animal between stages 16 and 17 of adult development were immunostained by the MOSA. At stage 16, the MOSA immunoreactivity is not detectable except for faint staining of dendrites in a few male-specific sensilla trichodea (Fig. 4, $A, C$, $E$ ). In pupae at late stage 16 , dendrites of most of the sensilla trichodea are stained, and the reaction product is identifiable in cell bodies as a few faint dots. At stage 17, dendrites and cell bodies are densely stained (Fig. $4, B, D$ ). Only at this late stage of adult development does the male-specific macroglomerular complex (MGC) first exhibit staining by the MOSA. The MOSA immunoreactivity seems to accumulate first in dendrites and then in cell bodies and axons. We could detect no gradient of MOSA immunoreactivity along the length of the antennal flagellum.

\section{Discussion}

Precursors of the ORCs in the disk epithelium

The OSA recognizes many, and apparently all, ORCs in both males and females, and the MOSA stains only male-specific antennal ORCs in pharate adult Manduca (Hishinuma et al., 1988). Early in larval-pupal-adult metamorphosis, the OSA recognizes a subset of the epithelial cells of the antennal imaginal disk in fifth-instar larvae. The immunoblot analysis showed that one species of antigens, of 59,000 Da apparent molecular weight, migrated to the same position in PAGE of extracts of both disk epithelial cells and the developing antennae of pupae and pharate adults.

The OSA immunoreactivity is found in most of the epithelial cells in the proximal two-thirds of the inner cell layer of the disk epithelium and in the proximal one-third of the peripodial membrane. In the developing antennae, clusters of ORCs in each annulus of the flagellum form 2 longitudinal rows. If a subpopulation of the disk epithelial cells is committed (as are hair mother cells) to become the ORCs, then the OSA immunostaining might reveal 2 longitudinal bands in the imaginal disk. We reconstructed serial sections of antennal imaginal disks, but no such bands were detected. This finding supports the hypothesis that no particular cells are predetermined to form bristles or hairs (Lawrence, 1966) and suggests that a subgroup of the OSA-immunoreactive cells is selected to become hair mother cells by undetermined contemporaneous factors (Lawrence, 1966). In Drosophila, expression of position-specific antigens that reflect dorsoventral regional differences within wing imaginal disks correlates only with the position of disk cells, rather than with their ultimate differentiation (Wilcox et al., 1981; Brower et al., 1984). From his morphological study, Eassa (1953) suggested that a proximal part of the peripodial membrane contributes to the formation of the head capsule. Our finding that the OSA immunoreactivity resides in the proximal one-third of the peripodial membrane suggests that the OSAimmunoreactive epithelial cells are capable of giving rise to the ORCs even though they generate head capsule, or that they actually contribute to the formation of antennal ORCs, contrary to Eassa's hypothesis. In the antennal imaginal disk of the fifthinstar larva, a gradient of differentiation similar to the posteroanterior movement of the "morphogenic furrow" in Drosophila eye disks (Melamed and Trujillo-Cenóz, 1975; Ready et al., 1976; Zipursky et al., 1984) is not detected. The OSA immunoreactivity in the disk epithelium is lost during the prepupal stage and is first detected again in the developing antenna 70 $\mathrm{hr}$ after the larval-pupal molt. ${ }^{3} \mathrm{H}$-Thymidine birthdating studies indicate that DNA synthesis in the ORCs during differentiative divisions (S phase) is finished by $60 \mathrm{hr}$ after the larvalpupal molt (Sanes and Hildebrand, 1976b). Therefore, around $70 \mathrm{hr}$ after the molt, the differentiative cell divisions of the ORCs are likely to be finished. Once they are postmitotic, the ORCs elaborate axons as one of the earliest events in the maturation of a sensillum (Wigglesworth, 1953; Sanes and Hildebrand, 1976b). The reappearance of the OSA immunoreactivity in the ORCs bearing axons, but not in cells lacking axons, confirms that the OSA immunoreactivity reappears in the ORCs only after the differentiative cell divisions.

The MOSA immunoreactivity in the disk epithelium is lost during the prepupal stage and is first detectable again in cells scattered in the antennal lumen immediately after the larvapupal molt. During the subsequent few days, these cells apparently multiply to form clusters of 20-30 cells. They lose the MOSA immunoreactivity between stages 4 and 7 and then disappear by stage 16 . The location of these cells in the antenna and their temporal existence suggest that they are developmental remnants. It remains to be determined, however, what relationship the antigen expressed in the disk epithelial cells bears to that in the cells scattered in the antennal lumen or in the pheromone-receptor cells.

\section{Developmental regulation of the expression of the OSA antigens}

On immunoblots the OSA binds to specific sets of the electrophoretic bands at various stages of metamorphic adult development. The immunoreactive bands of 53,000 and 59,000 Da apparent molecular weight are obtained from the disk epithelium; the $53,000,59,000$, and $66,000 \mathrm{Da}$ bands, from the antennal epithelium at stages $3-10$; and the $42,000,59,000$, and $66,000 \mathrm{Da}$ bands, from antennal epithelium at stages 12-18 (pharate-adult). The change in the OSA-immunoreactive bands between stages 10 and 12 coincides with degeneration of the " $t 2$ " cells of Sanes and Hildebrand (1976b). Immunocytochemical testing of the epithelium at stage 7 demonstrates, however, 
that those short-lived cells do not express the OSA antigens. By contrast with the $t 2$ cells, which are found at the apical half of the sensilla (Sanes and Hildebrand, 1976b), OSA-immunoreactive cell bodies are found at the base of the antennal epithelium. Their immunoreactive dendrites traverse the antennal epithelium toward the sensory hairs. This situation also describes the ORC somata and dendrites at stage 7 (Sanes and Hildebrand, 1976b). The changes in the OSA antigen species in immunoblots thus appear to occur in the antennal ORCs.

It is not yet known how the differences among the OSA antigens arise and how their expression is regulated during postembryonic development. Are they encoded by different genes, or are their differences generated by transcriptional, translational, or posttranslational modifications? The major changes in the expression of these antigens coincide with the changes in ecdysone titer in hemolymph (Bollenbacher et al., 1981). The titer of ecdysone decreases in the prepupal period when the OSA antigens become undetectable in the disk epithelium, increases very sharply at stage 3 when the antigens reappear in the ORCs in the antennal epithelium, and rapidly declines at stage 10 when the antigen species change. Ecdysone, which is one of the major steroid hormones in invertebrates (Riddiford and Truman, 1978; Granger and Bollenbacher, 1981), transcriptionally regulates the expression of several genes in a Drosophila cell line (Cherbas et al., 1977; Best-Belpomme et al., 1978; Savakis et al., 1980; Couderc et al., 1983; Savakis et al., 1984). If ecdysone is similarly involved in the regulation of the OSA antigens, one might hypothesize that the differential expression of each antigen recognized by the OSA during postembryonic development is regulated by ecdysone either via the expression of a family of genes, each of which encodes for one of the OSA antigens, or via induction of expression of a genc coding for an enzyme that modifies transcriptional or translational products.

\section{Toward the functions of the antigens}

In the preceding paper (Hishinuma et al., 1988), we showed that the OSA recognizes antigens associated with the cell membrane (and possibly on the cell surface), and that the OSA-positive axons form 2 bundles of fascicles in the antennal nerve (AN) distinct from a major mechanosensory bundle. Immediately after the ORCs become postmitotic, they send out axons that grow toward the ALs. In each annulus of the antennal flagellum, the olfactory axons aggregate into 2 nerves, one on each side, which then merge with the 2 branches of the AN that run in the lumen of the antenna (Sanes and Hildebrand, 1976a, b). On immunoblots, one of the membrane-associated glycoproteins $(66,000 \mathrm{Da})$ is not detected in extracts of the disk epithelium, which has only relativcly few axons associatcd with it (Sancs and Hildebrand, 1975), but is expressed in the axon-rich developing antennal epithelium. Furthermore, the 66,000 Da protein is greatly enriched in axons and axon terminals of the ORCs (Hishinuma et al., 1988). Because the OSA immunoreactivity is first detected in growing axons as well as in cell bodies, and may be a cell-surface component, and because no ORCs are stained before the outgrowth of axons, the $66,000 \mathrm{Da}$ protein is a candidate for the role of mediating fasciculation of the olfactory axons. A similar antigen expressed on axonal surfaces has been described in the leech nervous system (Hockfield and McKay, 1983; McKay et al., 1983). Alternatively, because the 66,000 Da protein is expressed in growing axons near their targets in the CNS, it is a candidate for a possible role in target recognition.
The antigen recognized by the MOSA is expressed at the late stage of adult development, when antennal responses to the female sex pheromones are first detectable by electroantennography. Previous studies in our laboratory used $E$-2-hexenal (a common volatile constituent of plants) and the female sex pheromones for electroantennogram studies of the functional maturation of the ORCs (Schweitzer et al., 1976). The antenna in both males and femlaes is similarly responsive to $E$-2-hexenal, but only the male antenna is responsive to the pheromones. $E$-2-Hexenal first elicited responses in the antenna at stage 15 , but only at stage 17 were responses to the pheromones first detectable. Therefore, the expession of the MOSA antigen in the pheromone-receptor cells in the male antenna corresponds to the period when the cells become responsive to the pheromones, but not to $E$-2-hexenal. The temporal coincidence between the expression of the MOSA antigen and the initial detection of the responses to the pheromones is consistent with the notion that the MOSA antigen might be functionally related to detection of the pheromones.

In summary, the cell-type-specific Mabs, the OSA, and the MOSA, which were first isolated on the basis of previous anatomical information about the sexually dimorphic olfactory system of Manduca, have proved to be useful molecular probes that reveal morphological and temporal phenomena not detected by conventional anatomical and physiological techniques. The temporal correlation between the expression of the antigens and events in the differentiation and maturation of the ORCs provokes interest in the regulation of the expression of the antigens and their functions. Studies under way focus on those issues.

\section{References}

Best-Belpomme, M., A. M. Courgeon, and A. Rambach (1978) $\beta$-Galactosidase is induced by hormone in Drosophila melanogaster cell cultures. Proc. Natl. Acad. Sci. USA 75: 6102-6106.

Bollenbacher, W. E., S. L. Smith, W. Goodman, and L. I. Gilbert (1981) Ecdysteroid titer during larval-pupal-adult development of the tobacco hornworm, Manduca sexta. Gen. Comp. Endocrinol. 44: 302306.

Brower, D. L., M. Wilcox, M. Piovant, R. J. Smith, and L. A. Reger (1984) Related cell-surface antigens expressed with positional specificity in Drosophila imaginal discs. Proc. Natl. Acad. Sci. USA 81: 7485-7489.

Cherbas, P., L. Cherbas, and C. M. Williams (1977) Induction of acetylcholinesterase activity by $\beta$-ecdysone in a Drosophila cell line. Science 197: 275-277.

Couderc, J. L., M. L. Sobrier, G. Giraud, J. L. Becker, and B. Dastugue (1983) $\Lambda$ ctin gene expression is modulated by ecdysterone in a Dro sophila cell line. J. Mol. Biol. 164: 419-430.

Eassa, Y. E. E. (1953) The development of imaginal buds in the head of Pieris brassicae Linn. (Lepidoptera). Trans. R. Entomol. Soc. Lond. 104: 39-50.

Granger, M. A., and W. E. Bollenbacher (1981) Hormonal control of insect metamorphosis. In Metamorphosis-A Problem in Developmental Biology, 2nd Ed., L. I. Gilbert and E. Frieden, eds., pp. 105137, Plenum, New York.

Hishinuma, A., S. Hockfield, R. McKay, and J. G. Hildebrand (1988) Monoclonal antibodies reveal cell-type-specific antigens in the sexually dimorphic olfactory system of Manduca sexta. I. Generation of monoclonal antibodies and partial characterization of the antigens. J. Neurosci. 8: 296-307.

Hockficld, S., and R. McKay (1983) Monoclonal antibodies demonstrate the organization of axons in the leech. J. Neurosci. 3: 369-375.

Köhler, G., and C. Milstein (1976) Derivation of specific antibodyproducing tissue culture and tumor lines by cell fusion. Eur. J. Immunol. 6: 511-519.

Laemmli, U. K. (1970) Cleavage of structural proteins during the assembly of the head of bacteriophage T4. Nature 227: 680-685. 
Lawrence, P. A. (1966) Development and determination of hairs and bristles in the milkweed bug, Oncopeltus fasciatus (Lygaeidae, Hemiptera). J. Cell Sci. 1: 475-498.

McKay, R. D. G., S. Hockfield, J. Johnsen, I. Thompson, and K. Frederiksen (1983) Surface molecules identify groups of growing axons. Science 222: 788-794.

Melamed, J., and O. Trujillo-Cenóz (1975) The fine structure of the eye imaginal disks in muscoid flies. J. Ultrastruct. Res. 51: 79-93.

Ready, D. F., T. E. Hanson, and S. Benzer (1976) Development of the Drosophila retina, a neurocrystalline lattice. Dev. Biol. 53: 217240.

Riddiford, L. M., and J. W. Truman (1978) Biochemistry of insect hormones and insect growth regulators. In Biochemistry of Insects, M. Rockstein, ed., pp. 307-357, Academic, New York.

Sanes, J. R., and J. G. Hildebrand (1975) Nerves in the antennae of pupal Manduca sexta Johanssen (Lepidoptera: Sphingidae). Wilhelm Roux' Arch. 178: 71-78.

Sanes, J. R., and J. G. Hildebrand (1976a) Structure and development of antennae in a moth, Manduca sexta. Dev. Biol. 51: 282-299.

Sanes, J. R., and J. G. Hildebrand (1976b) Origin and morphogenesis of sensory neurons in an insect antenna. Dev. Biol. 51:300-319.

Savakis, C., G. Demetri, and P. Cherbas (1980) Ecdysteroid-inducible polypeptides in a Drosophila cell line. Cell 22: 665-674.

Savakis, C., M. M. D. Koehler, and P. Cherbas (1984) cDNA clones for the ecdysonc-inducible polypeptide (EIP) mRNAs of Drosophila Kc cells. EMBO J. 3: 235-243.
Schweitzer, E. S., J. R. Sanes, and J. G. Hildebrand (1976) Ontogeny of electroantennogram responses in the moth, Manduca sexta. J. Insect Physiol. 22: 955-960.

Shorr, R. G., A. Lyddiatt, M. M. S. Lo, J. O. Dolly, and E. A. Barnard (1981) Acetylcholine receptor from mammalian skeletal muscle. Eur. J. Biochem. 116: 143-153.

Tolbert, L. P., S. G. Matsumoto, and J. G. Hildebrand (1983) Development of synapses in the antennal lobes of the moth Manduca sexta during metamorphosis. J. Neurosci. 3: 1158-1175.

Towbin, H., T. Staehelin, and J. Gordon (1979) Electrophoretic transfer of protcins from polyacrylamide gels to nitrocellulose sheets: Procedure and some applications. Proc. Natl. Acad. Sci. USA 76:43504354.

Vince, R. K., and L. I. Gilbert (1977) Juvenile hormone esterase activity in precisely timed last instar larvae and pharate pupae of Manduca sexta. Insect Biochem. 7: 115-120.

Wigglesworth, V. B. (1953) The origin of sensory neurones in an insect, Rhodnius prolixus (Hemiptera). Q. J. Microsc. Sci. 94:93-112.

Wilcox, M. D. L. Brower, and R. J. Smith (1981) A position-specific cell surface antigen in the Drosophila wing imaginal disc. Cell 25. 159-164.

Zipursky, S. L., T. R. Venkatesh, D. S. Teplow, and S. Benzer (1984) Neuronal development in the Drosophila retina: Monoclonal antibodies as molecular probes. Cell 36: 15-26. 